\title{
Cardiothoracic CT: one-stop-shop procedure? Impact on the management of acute pulmonary embolism
}

\author{
Pauline J. Abrahams-van Doorn • \\ Ieneke J. C. Hartmann
}

Received: 17 December 2010/Revised: 2 May 2011 / Accepted: 18 July 2011 /Published online: 6 August 2011

(C) The Author(s) 2011. This article is published with open access at Springerlink.com

\begin{abstract}
In the treatment of pulmonary embolism (PE) two groups of patients are traditionally identified, namely the hemodynamically stable and instable groups. However, in the large group of normotensive patients with PE, there seems to be a subgroup of patients with an increased risk of an adverse outcome, which might benefit from more aggressive therapy than the current standard therapy with anticoagulants. Risk stratification is a commonly used method to define subgroups of patients with either a high or low risk of an adverse outcome. In this review the clinical parameters and biomarkers of myocardial injury and right ventricular dysfunction (RVD) that have been suggested to play an important role in the risk stratification of PE are described first. Secondly, the use of more direct imaging techniques like echocardiography and $\mathrm{CT}$ in the assessment of RVD are discussed, followed by a brief outline of new imaging techniques. Finally, two risk stratification models are proposed, combining the markers of RVD with cardiac biomarkers of ischemia to define whether patients should be admitted to the intensive care unit (ICU) and/or be given thrombolysis, admitted to the medical ward, or be safely treated at home with anticoagulant therapy.
\end{abstract}

Keywords Pulmonary embolism $\cdot$ Risk stratification $\cdot$ CT . Cardiac biomarkers $\cdot$ Right ventricular dysfunction

\footnotetext{
P. J. Abrahams-van Doorn $(\triangle) \cdot$ I. J. C. Hartmann

Department of Radiology, Erasmus MC University Medical Center Rotterdam,

's-Gravendijkwal 230,

NL-3015 CE, Rotterdam, The Netherlands

e-mail: p.j.vandoorn@erasmusmc.nl
}

\section{Introduction}

Pulmonary embolism (PE) is a common and potentially fatal cardiovascular disorder. The clinical presentation of acute PE reveals a wide spectrum, ranging from mild dyspnea to cardiac arrest. The short-term mortality of PE also varies widely, ranging from less than $1 \%$ in hemodynamically stable patients with non-massive PE and no signs of right heart overload to over $90 \%$ in patients who present with cardiorespiratory arrest [1].

In the treatment of PE two groups of patients are traditionally identified. Given the high mortality risk in hemodynamically unstable patients, aggressive therapies such as thrombolytics, inotropic vasoactive drugs, embolectomy, or thrombus fragmentation are indicated [2-4]. In the large group of normotensive patients, anticoagulant treatment is started as the risk of an adverse event due to more aggressive treatment is considered higher than the relatively low risk of PE-related death.

Recent studies have demonstrated that up to $55 \%$ of normotensive patients with $\mathrm{PE}$ have asymptomatic right ventricular dysfunction (RVD). These normotensive patients with RVD have a higher risk of an adverse outcome with an estimated 30-day mortality directly related to PE between 3 and 10\% [5], and an absolute increase in early PE-related mortality of $4-5 \%$ [6], or a doubling of the risk of all-cause death during 3-month follow-up [7]. This is significantly higher than the early PE-related mortality in the overall group of normotensive patients $(1-7 \%)[8,9]$. It has been debated that normotensive PE patients with RVD might benefit from thrombolytic therapy $[10,11]$. In contrast, there may also be a subgroup of hemodynamically stable patients without RVD that have a very low risk of adverse outcomes and in whom early hospital discharge or even home treatment may be considered. 
Risk stratification is a frequently used method to identify subgroups of patients that may benefit from different diagnostic or treatment methods based on a different risk profile. In this review, we discuss the parameters that are currently proposed for prognostic stratification of patients with acute PE. These parameters can, from a practical point of view, be classified into three groups: the first group consists of clinical parameters that can be immediately obtained at bedside, mainly to assess hemodynamic status and distinguish high-risk from non-high-risk PE patients. The second group contains biomarkers of myocardial injury, and finally, markers of RVD make up the third group. Both cardiac biomarkers and markers of RVD can be used to further stratify non-high-risk PE patients into intermediate- and low-risk subgroups [3]. Based on the literature currently available, a potential risk stratification strategy is proposed. The benefit of this and other risk stratification strategies, however, is currently under investigation and has to be demonstrated in prognostic studies before implementation in clinical practice can be recommended. Before we discuss the parameters that are currently considered for clinical risk stratification of patients with PE, we will first expound the pathophysiology of PE to understand the broad clinical spectrum of PE.

\section{Pathophysiology of PE}

PE can lead to RVD and eventually cardiac failure due to several mechanisms (Fig. 1). At first, PE leads to pulmonary artery (PA) obstruction, which increases the pulmonary vascular resistance. This, in turn, results in an increase in right ventricular (RV) afterload, causing RVD, together resulting in a decreased RV output. At the same time the clot induces the release of neurohumoral substances stimulating arterial vasoconstriction and hypoxemia, thereby increasing pulmonary vascular resistance and RV afterload. Eventually, when RVD is the result of the PE, an increase in RV volume and RV dilatation can be the next step. Because the heart is contained within the pericardium, an increase in RV volume also leads to a shift of the interventricular septum to the left, decreasing the left ventricular (LV) preload and output. In the end, this leads to a decreased cardiac output.

As a consequence of the increased RV afterload, the increased RV wall tension, and increased oxygen demand of the RV, the coronary perfusion of the RV will decrease, leading to ischemia. This in turn stimulates further RVD. Finally, pre-existent cardiopulmonary disease further contributes to the severity of the hemodynamic alterations.

The large differences in outcome in PE are assumed to be related both to the embolus burden and the cardiopulmonary status [12]. Wood et al. proposed a relationship between the severity of the embolic event, characterized by both the embolus size and the cardiopulmonary function, and the mortality in patients with PE. They state that the combination of the embolus size and the cardiopulmonary function leading to shock seems to be associated with a mortality of approximately $30 \%$. Furthermore, a slightly further increase in severity would lead to cardiac arrest, having a mortality of at least $70 \%$, and eventually lead to sudden death. However, it remains unclear which exact combinations of embolus load
Fig. 1 Pathophysiology of pulmonary embolism. Due to obstruction of the pulmonary vascular bed, increased vascular resistance, and increased RV afterload, PE can lead to RVD. RVD can have several consequences. First, it can decrease RV output. Second, RVD may result in a decrease of LV preload and output, caused by an increased RV volume and dilatation, eventually leading to decreased cardiac output. Finally, RVD can cause decreased coronary perfusion, leading to ischemia, which in turn results in a further increase of RVD. RV = right ventricle, $\mathrm{PE}=$ pulmonary embolism, RVD = right ventricular dysfunction, $\mathrm{LV}=$ left ventricle, $\mathrm{PA}=$ pulmonary artery

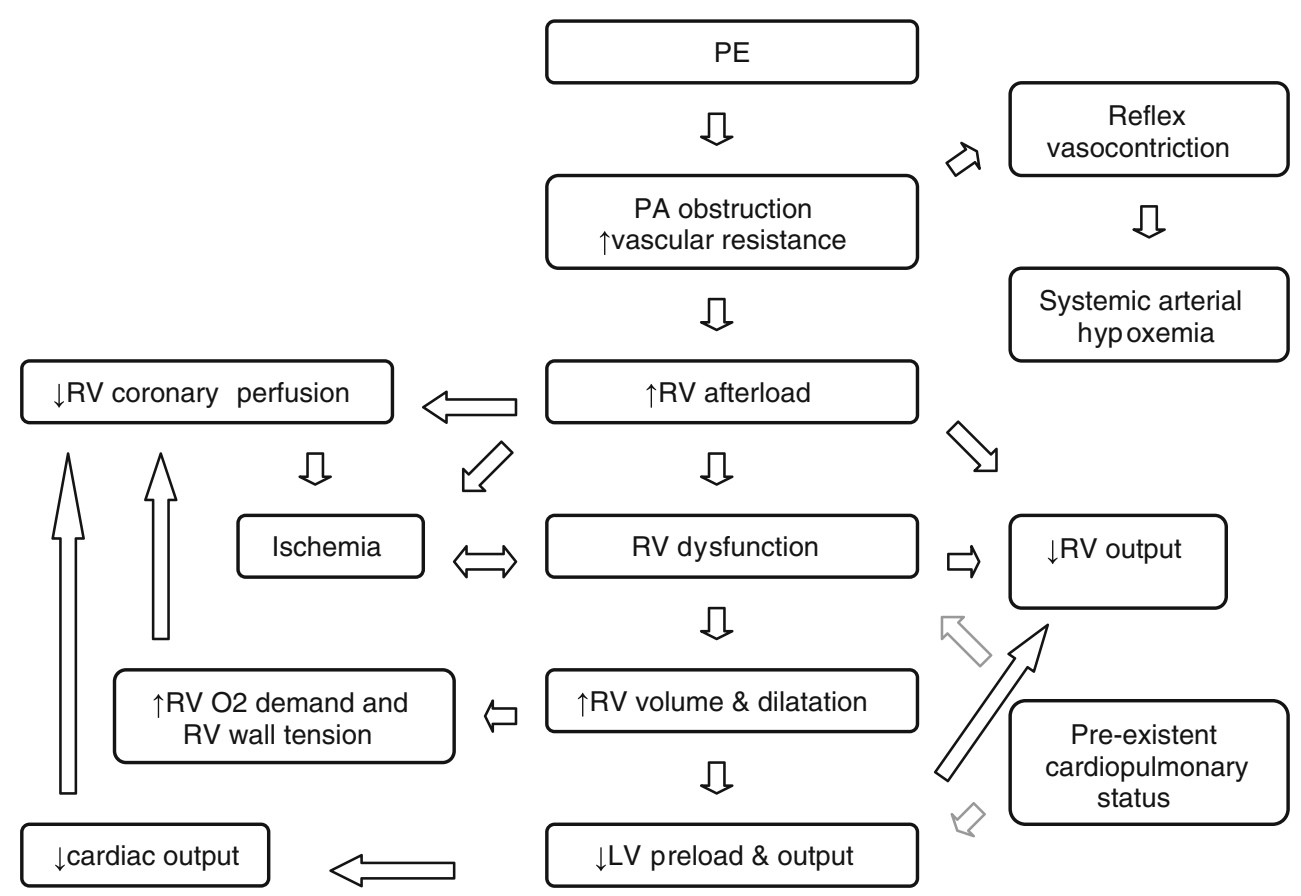


and cardiopulmonary status may lead to hemodynamic instability and consequently an exponential increase in mortality rates in normotensive patients with PE. Therefore, it might be of interest to know if there are any predictors that account for this rapid increase in mortality. It is thought that in the group of normotensive patients RVD is an important indicator of an increase in mortality [12].

\section{Clinical parameters}

The most important clinical prognostic factors for PE are hypotension and shock [13]. As mentioned before, these patients with hypotension and shock are at high risk, having a short-term mortality of approximately $30 \%$ [12]. Because of hemodynamic instability, computed tomography (CT), pulmonary angiography or scintigraphy usually is not performed in the immediate diagnostic workup, and the diagnosis is usually made on the basis of high clinical suspicion and signs of RV failure on echocardiography [3].

However, clinical factors may not only be useful for the initial identification of potentially fatal PE patients, but some of them can also be used to identify patients at the other end of the clinical spectrum, namely those that are at low-risk of an adverse outcome. A commonly used prognostic model is the Pulmonary Embolism Severity Index (PESI) based on 11 patient factors that are all independently associated with short-term mortality and non-fatal adverse outcomes (Table 1) [7, 14]. The PESI was developed to identify low-risk patients who may be potential candidates for outpatient treatment or early hospital discharge.

\section{Cardiac biomarkers}

Cardiac biomarkers are currently used in the diagnostic workup of acute chest pain and dyspnea. However, there is increasing evidence that they may also be relevant in PE risk stratification [15]. Cardiac biomarkers can be subdivided into two major groups: markers of RVD, particularly brain natriuretic peptides, and markers of myocardial injury, of which troponins are the most important representatives.

\section{Brain natriuretic peptides}

Brain natriuretic peptides (BNP) or N-terminal proBNP (NTproBNP) are cardiac biomarkers released with increased myocardial stretch. There is increasing evidence that the BNP and NT-proBNP levels are directly related to the severity of RVD $[16,17]$. In a systematic review, Sanchez et al. found a relative risk for predicting 30-day mortality of 9.5 for BNP
Table 1 Pulmonary embolism severity index

\begin{tabular}{ll}
\hline Predictors & Score \\
\hline Age & Age, in years \\
Male sex & 10 \\
Cancer & 30 \\
Heart failure & 10 \\
Chronic lung disease & 10 \\
Pulse $\geq 110 /$ min & 20 \\
Systolic blood pressure $<100 \mathrm{mmHg}$ & 30 \\
Respiratory rate $\geq 30 / \mathrm{min}$ & 20 \\
Temperature $<36^{\circ}$ & 20 \\
Altered mental status* & 60 \\
Arterial oxygen saturation $<90 \% * *$ & 20 \\
\hline
\end{tabular}

The pulmonary embolism severity index (PESI) consists of 11 indepent predictors of short-term mortality in patients with acute pulmonary embolism [14]. By adding the patient's age in years and the applicable predictor, a total score is obtained. Based on this total score patients are classified into one of the five risk classes: $\leq 65$ class I, 66 85 class II, 86-105 class III, 106-125 class IV, $>125$ class V. Classes I an II are defined as low risk; classes III-V are defined as higher risk

*Defined as disorientation, lethargy, stupor or coma

**With and without the administration of supplemental oxygen

and 5.7 for pro-BNP in patients with proven PE [18]. Although the negative predictive value (NPV) of BNP/NTproBNP is very high $(94-100 \%)$, the positive predictive value (PPV) is rather low (12-26\%) [3]. Consequently, these biomarkers seem especially useful to identify patients with PE who are at low risk of an adverse outcome.

\section{Cardiac troponins}

Elevated levels of troponins (either I or T) are found in patients with acute myocardial infarction due to injury of myocardial cells [19]. Troponin- $\mathrm{T}$ is released when RV ischemia is present. Elevated troponin T levels were found in $32 \%$ of patients with massive and moderate PE, but not in patients with mild PE [20]. Furthermore, Sanchez et al. revealed a strong relationship between elevated troponin T levels and 30-day mortality [18]. Although elevated troponin levels are associated with increased early mortality, the PPV is low (12-44\%) [3]. In contrast, normal troponin T levels revealed a high NPV of 99$100 \%$ for both fatal and non-fatal adverse events [3]. Troponin levels may therefore be useful either as a marker of low risk of an adverse outcome when they are normal or as a marker of high risk when they are elevated.

\section{Other biomarkers}

The latest biomarkers studied include the fatty acid binding proteins (FABPs), which have been proposed as more 
sensitive markers of myocardial injury than troponin $\mathrm{T}$ and NT-proBNP [21], and are associated with several adverse outcomes, including shock, intubation and cardiopulmonary resuscitation [22]. D-dimer is a biomarker of fibrinolysis and inflammation, and is elevated in the presence of an acute clot. In patients with PE, plasma D-dimer levels were found to be higher in patients who died of PE than in those who survived [23]. Growth differentiation factor 15 (GDF$15)$ is a cytokine released in the heart in response to ischemia or pressure overload. In patients with acute PE, GDF-15 was an independent predictor of a complicated 30day course and of long-term mortality [24].

Although all these relatively new biomarkers can be considered of additional value in relation to the prognosis of PE, they have not yet been evaluated in prospective studies, and their potential role as an independent predictor of adverse outcome in PE patients still needs to be determined.

\section{Imaging assessment in RVD}

As mentioned before, RVD is an important prognostic indicator in patients with PE. Whereas cardiac biomarkers are an indirect parameter of RVD, it can be more directly assessed using imaging methods such as echocardiography and computed tomography angiography (CTA).

\section{Echocardiography}

Echocardiography is widely used for the assessment of RVD in patients with PE [25]. Although a generally accepted definition of RVD in echocardiography is lacking [3], echocardiographic findings that are helpful and frequently used in the diagnosis of RVD are: RV dilatation, RV free wall hypokinesis, paradoxal septal wall motion, PA dilatation, a systolic pressure gradient between RV and LV $>30 \mathrm{mmHg}$, and PA flow acceleration time $<80 \mathrm{~ms}[10,18$, 26, 27]. RV dilatation as a marker of RVD is mostly determined by the ratio of RV end-diastolic diameter/LV enddiastolic diameter, with a ratio $\geq 0.6-1.0$ considered abnormal according to several authors [18]. In the International Cooperative Pulmonary Embolism Registry (ICOPER) RV hypokinesis was revealed to be an independent predictor of 30-day mortality in normotensive patients (hazard ratio $1.94,95 \%$ CI $1.23-3.06$ ) [28]. It was already found to double the risk of death within the ensuing 3 months in a study published by the same group [7]. In a retrospective analysis by Sukija et al. paradoxal septal wall motion increased the in-hospital mortality in patients with acute PE [29]. The other indirect signs of RVD, i.e., dilatation of the right PA, a systolic pressure gradient between the RV and right atrium (RA) $>30 \mathrm{mmHg}$, and a PA flow acceleration time $<80 \mathrm{~ms}$, have also been used by several authors in defining RVD [15, 26, 30-32].

\section{Computed tomography}

While pulmonary angiography has long been considered the gold standard in the diagnosis of PE, nowadays computed tomography angiography (CTA) is the first imaging method of choice in the workup of patients with suspected PE [33]. Besides diagnosing PE by direct visualization of the thrombus, CTA can also be helpful in the risk stratification of patients with PE. As with echocardiography the RV/LV diameter ratio as a parameter of RVD can easily be assessed on CT [3]. On axial views a $\mathrm{RV} / \mathrm{LV}$ ratio $\geq 0.9-1.5$ has been shown to be directly correlated with RVD and adverse outcome [34]. The PPV is low $(10 \%)$, but a RV/LV ratio $<1$ has a high NPV $(100 \%)$ [35]. Two examples of patients with extensive PE and RVD are given in Figs. 2 and 3. One patient suffered cardiac arrest and died shortly after; the other patient was hemodynamically stable at presentation.

The additional value of a four-chamber view in the assessment of the RV/LV ratio has been mentioned, but these data are inconclusive [36-39]. These contradicting results, together with the necessity of additional software and extra time in order to obtain a RV/LV ratio on fourchamber view, make assessment on the axial views the preferred method these days.

When the results of studies on the prognostic role of RVD assessed on echocardiography are taken together with those assessed on CT, the relative risk of RVD for predicting death is 2.4 [18]. Furthermore, RVD may also predict poor pulmonary clot resolution at 6 months, and a higher incidence or venous thromboembolism (VTE) recurrence was demonstrated when RVD was present [40].

\section{Controversial markers of RVD}

Several other parameters in predicting RVD on CTA have been mentioned, such as dilatation of the superior vena cava (SVC) and azygos vein (AV), resulting from an acute increase in blood volume and pressure in the RA. A study of Ghaye et al. in patients with severe PE revealed significantly different diameters of the SVC and AV between survivors and non-survivors, but only the AV diameter was found to predict mortality [41]. Another study not only demonstrated the AV diameter as a predictor of mortality in acute PE, but the SVC diameter was also significantly correlated to mortality [42]. A more recent publication showed the AV diameter to be significantly increased in the severe PE group compared with the nonsevere PE group, whereas no statistically significant difference in diameter of the SVC was present [43]. The 

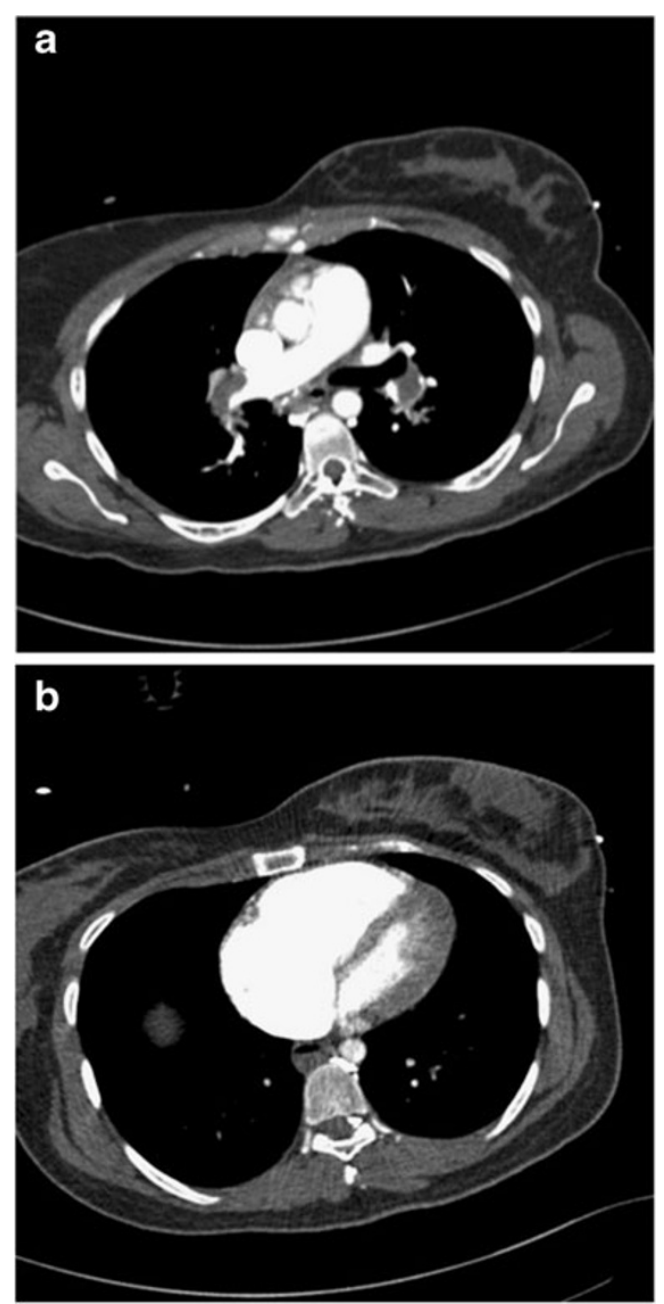

Fig. 2 CTPA of a 31-year-old female patient who was reanimated. The 1-mm axial reconstructions demonstrate bilateral central pulmonary emboli with dilatation of the main pulmonary artery (a) and massive right-sided cardiac dilatation with compression of the left ventricle (b). The patient died shortly after as a result of massive cerebral ischemia

dilatation of the AV has so far been revealing the strongest correlation with adverse outcome in PE patients, but whether there is an additional value as compared to other independent predictors of adverse outcome in PE has not been determined yet.

Because the increase in blood volume and pressure in the RA and eventually in the RV can also cause backflow in the inferior vena cava (IVC), enlargement of the diameter of the PA, and straightening, bowing or paradoxal movement of the interventricular septum, these parameters have been the subject of several studies as a potential marker of RVD. However, these studies show discrepancies regarding the potential of these markers as an independent predictor of both fatal and non-fatal adverse outcomes [42, 44-46]. Although, for instance, the diameter of the PA has long been used as a parameter of RVD in echocardiography, Van der Meer et al. did not find a significant relationship between PA to ascending aorta diameter ratio and PErelated mortality [35].

If and how these parameters can be used in a risk stratification model has to be the subject of future prospective research.

Thrombus load

As already stated, the clinical spectrum of PE and the large differences in outcome are considered to be related to both thrombus load and cardiopulmonary status. Because of its excellent clot imaging, CTA can also be used for the quantification of the thrombus load in the pulmonary vascular tree, for which four different scoring systems have been proposed. A detailed discussion of these individual scoring systems is beyond the scope of this article. The modified Walsh and the Miller scores, which are both angiographic scores adapted to the needs of spiral CT, quantify the severity of pulmonary obstruction [47]. Both scores, however, do not differentiate between partial and complete obstruction of a pulmonary vessel. The more recent scores proposed by the groups of Qanadli and Mastora, which were primarily developed for CTA, not only give information about thrombus load, but also about the degree of obstruction $[48,49]$.

Despite its excellent clot imaging, the literature shows mixed results in the usefulness of the PA obstruction index as a predictor of RVD and mortality. Qanadli et al. found that a CT obstruction score of $40 \%$ or greater correlates well with RV dilatation by identifying more than $90 \%$ of the patients with PE and RV dilatation [49]. These results were confirmed in the study of Van der Meer et al., reporting an 11.2-fold increased risk of dying of $\mathrm{PE}$ for patients with an obstruction index of $40 \%$ or higher [35]. In a more recent study of Bazeed et al. a significant difference in the PA obstruction index of more than $50 \%$ between survivors and non-survivors of PE was present [50]. On the contrary, no association between clot burden and mortality was revealed by others $[42,44,46]$. Therefore, based on the current literature, there is insufficient support for a vascular obstruction score to be used in a risk stratification system of patients with PE.

\section{Other biomarkers}

New CT techniques: ECG-gated CTA and dual-source CT

Recent developments in CT might be able to further help us in the risk stratification of PE. ECG-gated CTA gives the possibility for dynamic and functional cardiac assessment [51]. Information can be obtained about the ejection fraction, $\mathrm{RV} / \mathrm{LV}$ volumes ratio, $\mathrm{RV}$ wall motion abnormality, 
Fig. 3 CTPA performed in a 22year-old male patient complaining of progressive dyspnea and chest pain, revealing extensive pulmonary emboli including the main pulmonary artery with dilatation of the main pulmonary artery (a, diameter $33 \mathrm{~mm}$ ) and the right side of the heart (b). The wedge-shaped subpleural consolidation (c) is caused by pulmonary infarction (d, pulmonary window setting). Because of the extensive load and the right ventricular dysfunction at $\mathrm{CT}$, the patient was admitted to the ICU for thrombolytic treatment. The patient became hypotensive shortly after arrival but recovered quickly after treatment. The follow-up CTPA after 1 week shows clearance of thrombus from the main pulmonary artery with normalization of its diameter (e, diameter $25 \mathrm{~mm}$ ) and normalization of the right ventricle. There is still extensive thrombus present on both sides
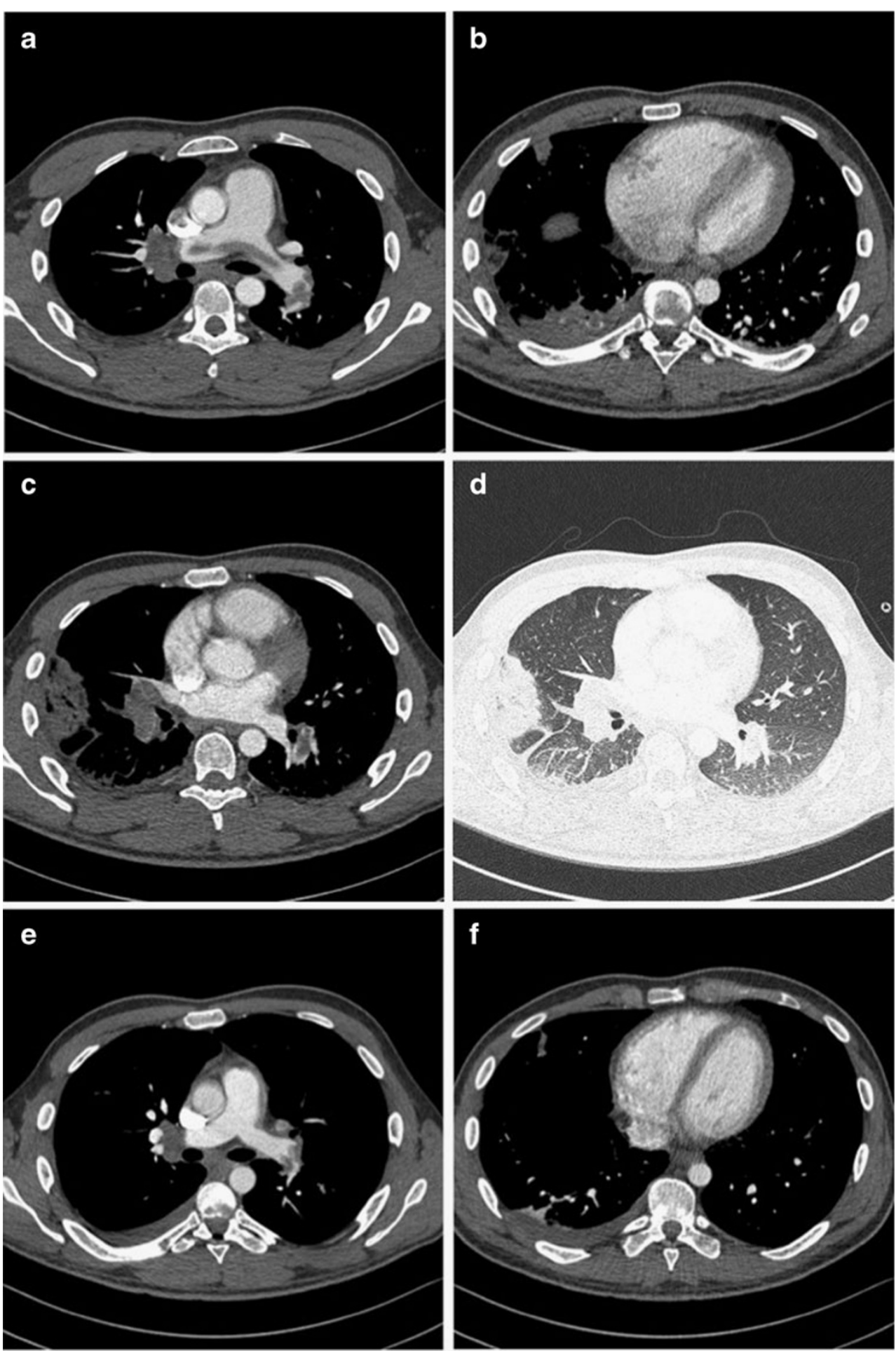

foramen ovale patency (right to left shunt), and intracardiac thrombus [45, 52], information that is currently provided by echocardiography. By using dedicated three-dimensional software for post-processing, obtaining these measurements does not have to be time-consuming, making it potentially useful in the acute clinical setting. Dual-source CT can be used in the diagnosis of PE in several ways. Using highpitch dual spiral protocols, the so-called flash technique, it is possible to scan the complete chest in less than a second [53], which is particularly helpful to obtain diagnostic quality in dyspneic patients. It can also easily be used for functional assessment of the heart using ECG gating. In addition, by using the dual-energy technique, it not only gives direct information on thrombus load, but also functional information on lung perfusion can be obtained at the same time $[54,55]$. This perfusion information can be 
Table 2 Prinicipal markers considered useful for risk stratification in acute pulmonary embolism [3]

$\mathrm{RVD}=$ right ventricular dysfunction

$\mathrm{RV}=$ right ventricle

$\mathrm{CT}=$ computed tomography

$\mathrm{BNP}=$ brain natriuretic peptide
Clinical markers
Shock
Hypotension

Markers of RVD

RV dilatation, hypokinesis, or pressure overload on echocardiography

RV dilatation in CT

BNP of NT-proBNP elevation

Elevated right heart pressure at right heart catheterization

Markers of myocardial injury Cardiac troponins useful, as small subsegmental emboli, compared to large non-occlusive emboli, can more easily result in complete obstruction of the pulmonary vascular bed and therefore may have a significant effect on the hemodynamic status. More research in this area needs to be done to prove its additional value in the risk stratification of PE.

Computer-assisted detection (CAD) software has been developed for the assessment of CTA in PE. First, CAD can be used as a second reader for improvement of the detection of peripheral PE [56]. Second, CAD may also be utilized for automated quantification of thrombus load [33], which is likely to lead to a significant reduction of the assessment time and therefore making evaluation of the thrombus load better applicable in the clinical setting. The incorporation of a CAD system was found to significantly improve assessment of PE severity. This was accomplished by increasing the observer's sensitivity and correctly raising the percentual embolic occlusion of the pulmonary arterial bed. Consequently, this better accuracy of the severity assessment resulted in improved agreement on the risk stratification of PE patients [57].

\section{Potential risk strategies}

In patients with PE, risk stratification models can be used to identify both patients who are either at high risk or at low risk of an adverse outcome after PE. Based on evidence currently available, the European Society of Cardiology (ESC) has defined principle markers useful for risk stratification in acute PE (Table 2) [3]. Of these, markers of RVD and cardiac biomarkers are likely to be the most attributable to a potential risk stratification system.

Several potential risk stratification models have been proposed. In some of these recommended algorithms markers of RVD and cardiac biomarkers were taken into consideration [3, 5, 15, 58]. Some authors combined cardiac biomarkers and echocardiography, selecting patients initially based on levels of BNP or troponin and secondly on signs of RVD on echocardiography [15]. In other strategies, it is proposed that imaging techniques like CTA or echocardiography should be used as a first step to establish the presence of RVD in a selected group of patients, namely those with an intermediate to high PESI
Estimated prevalence (\%)
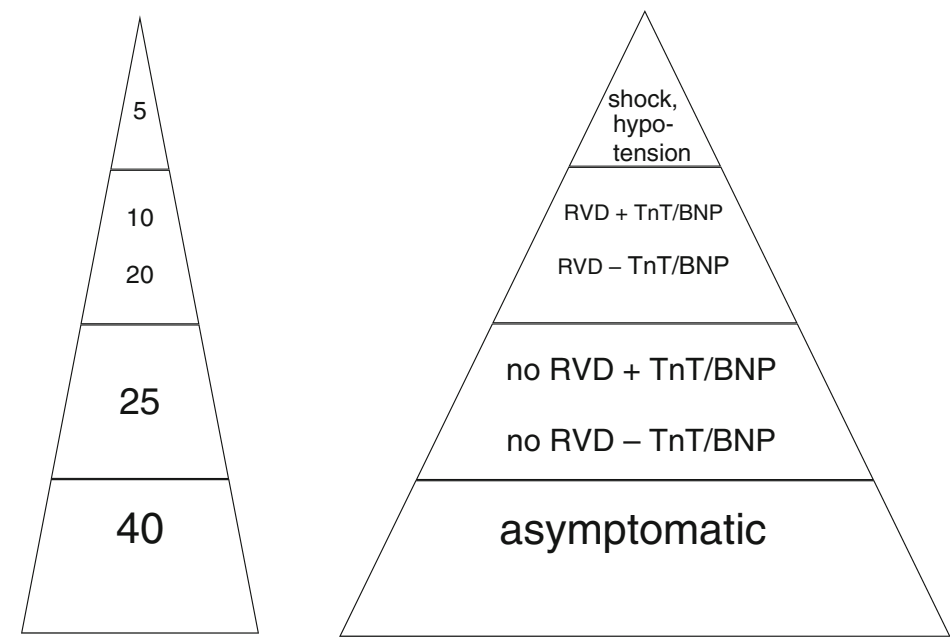

Estimated mortality (\%)

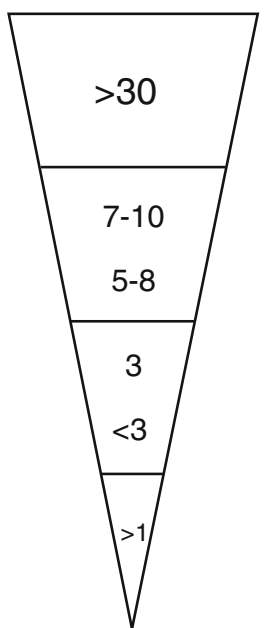

Fig. 4 The estimated prevalence and mortality of different risk factors in patients with pulmonary embolism (adapted from Becattini et al. 2008) [5]. $\mathrm{RVD}=$ right ventricular dysfunction, $\mathrm{TnT}=$ troponin $\mathrm{T}, \mathrm{BNP}=$ brain natriuretic peptide 
a
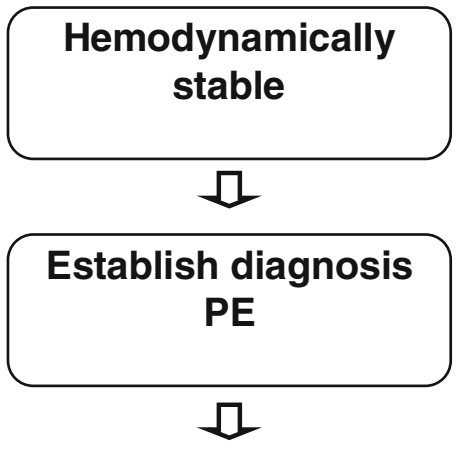

\section{RV function:}

Echocardiography, MDCT, BNP levels

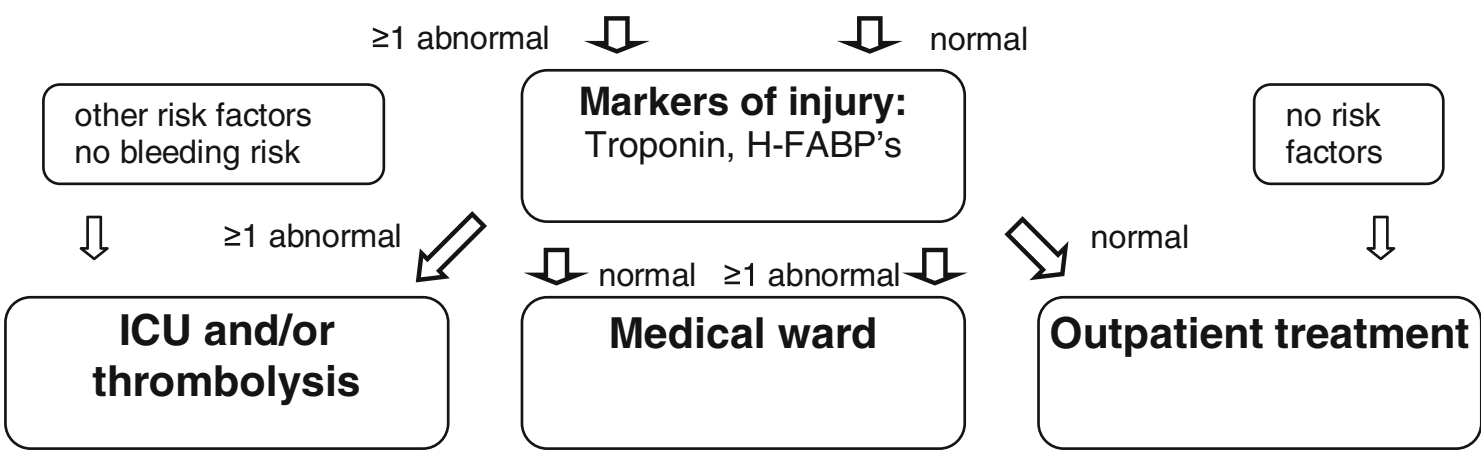

b
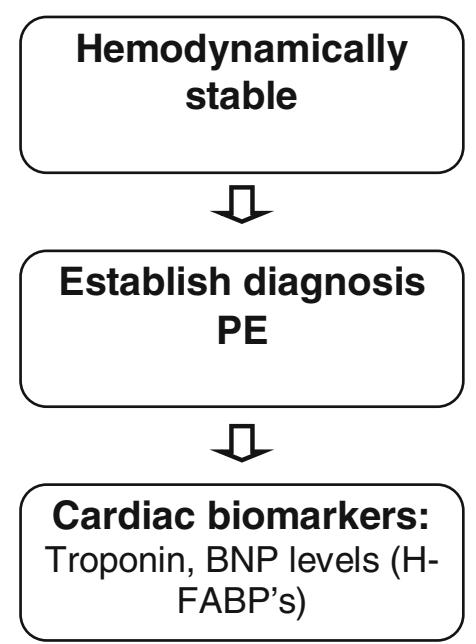

other risk factors no bleeding risk

I) $\geq 1$ abnormal

ICU and/or thrombolysis
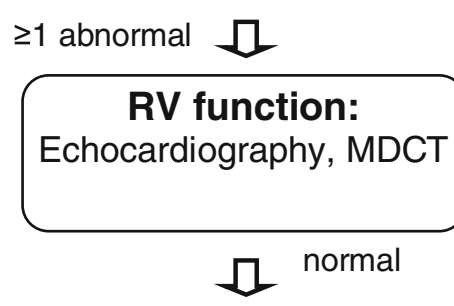

Medical ward no risk factors normal

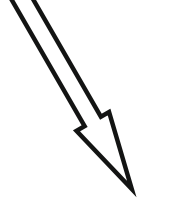

Outpatient treatment 
4 Fig. 5 a The clinical management of hemodynamically stable patients in pulmonary embolism. After establishing the diagnosis of PE, RV function should be determined, followed by the markers of myocardial injury. If there are signs of RVD and an elevation of troponin or fatty acid binding proteins, thrombolysis could be the next step. If there is only RVD or an elevation in the markers of myocardial injury, then the patient could be admitted to the medical ward. Outpatient treatment is the option in the absence of RVD or signs of myocardial injury. b After establishing the diagnosis of PE, the cardiac biomarkers are first taken into consideration. Outpatient treatment can only be considered if the cardiac biomarkers reveal no abnormalities. If these biomarkers are abnormal, RV function should be monitored. In case of RVD, only two options are left: admission to the medical ward in the absence of RVD or admission to ICU in combination with thrombolysis in the presence of RVD. $\mathrm{PE}=$ pulmonary embolism, $\mathrm{BNP}=$ brain natriuretic peptides, H-FABP's= fatty acid binding proteins, $\mathrm{RV}=$ right ventricle, $\mathrm{RVD}=$ right ventricular dysfunction, $\mathrm{MDCT}=$ multidetector computed tomography, ICU=intensive care unit

score and increased troponin levels [58]. Since CTA is the first imaging modality of choice in the diagnosis of PE in hemodynamically stable patients [33], additional information on RVD is obtained simultaneously and can therefore easily be incorporated in a risk stratification strategy. The clinical relevance and therapeutic consequence of any of these strategies, however, have so far not been evaluated in prospective studies, and their clinical value, therefore, remains uncertain.

Becattini et al. published a scheme in 2008 in which the prevalence of different features in patients with $\mathrm{PE}$ (asymptomatic, no RVD on ultrasound, RVD on ultrasound \pm increased troponin levels, hypotension and shock) was related to their mortality [5]. By introducing $\mathrm{CT}$ and the cardiac biomarkers defined by the ESC [3] in this scheme, we might be able to identify subgroups of hemodynamically stable patients that are either at high or low risk of both fatal and non-fatal adverse outcome (Fig. 4).

In the clinical management of hemodynamically stable patients, there are several options. Depending on the risk stratification, the management may range from outpatient treatment to admission to intensive care unit (ICU) and/or thrombolysis (Figs. 5a and b) [3, 59, 60]. There are currently no data available on whether thrombolysis and/or observation in the ICU is the best treatment option for high-risk PE patients, i.e., with $\mathrm{RVD}$, in the hemodynamically stable group. The results of an ongoing study on the benefit of thrombolysis in this high-risk PE group, including the patients that also have abnormal troponin levels (ClinicalTrials.gov NCT 00639743), will provide data that can be used in future risk stratification and treatment of this patient group. As mentioned before, whether outpatient treatment in low-risk patients is a good alternative to the initial conventional in-hospital i.v. heparin treatment for at least 5 days is currently under investigation (OTPE trial).

We propose two different risk stratification models in hemodynamically stable PE patients based on Becattini's proposed clinical management strategy and the revised Becattini prevalence scheme (Fig. 4). In Becattini's management proposal based on risk stratification, markers of dysfunction (ultrasound, MDCT, BNP levels) should be assessed first in hemodynamically stable patients with proven PE [5]. If normal, the patient can be admitted to a medical ward. If at least one marker of dysfunction is abnormal, markers of cardiac injury should be assessed (troponin, H-FABPs). Only patients in whom these markers are also abnormal should be admitted to the ICU.

The first proposed model (Fig. 5a) closely resembles Becattini's strategy. The main difference is the inclusion of other risk factors such as bleeding risk, and treatment with thrombolysis and outpatient treatment in the algorithm. Outpatient treatment can be a good option if both RVD and signs of myocardial injury are absent. In the second model (Fig. 5b), the cardiac biomarkers troponin, BNP and/or FABP are taken into consideration before assessing the presence of RVD by either CT or echocardiography. In this model, outpatient treatment can only be considered if the cardiac biomarkers reveal no abnormalities. If these biomarkers are abnormal, two options are left: admission to the medical ward when signs of RVD are absent or admission to the ICU in combination with thrombolysis if RVD is present. As CT is the first imaging method of choice in the diagnosis of acute PE and currently considered the gold standard for $\mathrm{PE}$, assessment of RV function on $\mathrm{CT}$ is a practical first step and should be evaluated in every patient with PE at CT. Cardiac echocardiography in general has a lower availability and has no proven additional value once $\mathrm{CT}$ has been performed. Laboratory testing including cardiac biomarkers should be obtained in every patient with suspected PE or acute cardiac disease. We think that this combination of CT and blood tests is the easiest strategy, most time-efficient, and probably most cost-effective. However, like the risk strategies proposed by other authors, these strategies need further prospective validation before they can be implemented in clinical practice.

\section{Summary}

In the large group of normotensive patients with PE, there seems to be a subgroup of patients with an increased risk of an adverse, even lethal, outcome, which might benefit from more aggressive therapy than the current standard therapy with anticoagulants. Risk stratification is a commonly used method to define subgroups of patients with either a high or low risk of an adverse outcome. Clinical parameters, biomarkers, or imaging markers of myocardial injury and RVD have been suggested to play an important role in the risk stratification of PE. In this overview we propose two risk stratification models combining parameters that have 
already been shown to be independent predictors of adverse outcomes, namely markers of RVD, either assessed with $\mathrm{CT}$, echocardiography or BNP, and cardiac biomarkers of ischemia to stratify patients into those that should be admitted to the ICU and/or be given thrombolysis, admitted to the medical ward, or can be safely treated at home with anticoagulant therapy. Like the risk strategies previously proposed by other authors, these strategies need further prospective validation before they can be implemented in clinical practice. New developments in CT, such as ECGgated CTA and dual-source CT, have the potential to further help us in the risk stratification of PE as these new techniques give us the possibility to obtain both functional and dynamic information at the same time in patients with suspected PE.

Open Access This article is distributed under the terms of the Creative Commons Attribution Noncommercial License which permits any noncommercial use, distribution, and reproduction in any medium, provided the original author(s) and source are credited.

\section{References}

1. Kurkciyan I et al (2000) Pulmonary embolism as cause of cardiac arrest: presentation and outcome. Arch Intern Med 160(10):15291535

2. Kearon C et al (2008) Antithrombotic therapy for venous thromboembolic disease. Chest $133(6$ suppl):454S-545S

3. Members ATF et al (2008) Guidelines on the diagnosis and management of acute pulmonary embolism. Eur Heart J 29 (18):2276-2315

4. Meneveau M (2010) Therapy for acute high-risk pulmonary embolism: thrombolytic therapy and embolectomy. Curr Opin Cardiol 25(6):560-567

5. Becattini C, Agnelli G (2008) Predictors of mortality from pulmonary embolism and their influence on clinical management. Thromb Haemost 100(5):747-751

6. ten Wolde $\mathrm{M}$ et al (2003) Brain natriuretic peptide as a predictor of adverse outcome in patients with pulmonary embolism. Circulation 107(16):2082-2084

7. Goldhaber SZ, Visani L, De Rosa M (1999) Acute pulmonary embolism: clinical outcomes in the International Cooperative Pulmonary Embolism Registry (ICOPER). Lancet 353 (9162):1386-1389

8. Douketis JD (2001) Prognosis in pulmonary embolism. Curr Opin Pulm Med 7(5):354-359

9. Nijkeuter $\mathrm{M}$ et al (2007) The natural course of hemodynamically stable pulmonary embolism: clinical outcome and risk factors in a large prospective cohort study. Chest 131(2):517-523

10. Konstantinides S (2008) Acute pulmonary embolism. N Engl J Med 359(26):2804-2813

11. Konstantinides S et al (1997) Association between thrombolytic treatment and the prognosis of hemodynamically stable patients with major pulmonary embolism: results of a multicenter registry. Circulation 96(3):882-888

12. Wood KE (2002) Major pulmonary embolism. Chest 121(3):877905

13. Aujesky D, Hughes R, Jiminéz D (2009) Short-term prognosis of pulmonary embolism. J Thromb Haemost 7(s1):318-321
14. Aujesky D et al (2005) Derivation and validation of a prognostic model for pulmonary embolism. Am J Respir Crit Care Med 172 (8):1041-1046

15. Konstantinides S (2005) Pulmonary embolism: impact of right ventricular dysfunction. Curr Opin Cardiol 20(6):496-501

16. Krüger S, Merx MW, Graf J (2003) Utility of brain natriuretic peptide to predict right ventricular dysfunction and clinical outcome in patients with acute pulmonary embolism. Circulation 108(13): 94

17. Tulevski II et al (2001) Increased brain natriuretic peptide as a marker for right ventricular dysfunction in acute pulmonary embolism. Thromb Haemost 86(5):1136-1342

18. Sanchez $O$ et al (2008) Prognostic value of right ventricular dysfunction in patients with haemodynamically stable pulmonary embolism: a systematic review. Eur Heart J 29(12):1569_ 1577

19. Thygesen K, Alpert JS, White HD (2007) Universal definition of myocardial infarction. J Am Coll Cardiol 50(22):2173-2195

20. Giannitsis E et al (2000) Independent prognostic value of cardiac troponin $\mathrm{T}$ in patients with confirmed pulmonary embolism. Circulation 102(2):211-217

21. Kaczyñska A et al (2006) Plasma heart-type fatty acid binding protein is superior to troponin and myoglobin for rapid risk stratification in acute pulmonary embolism. Clin Chim Acta 371 (1-2):117-123

22. Puls M et al (2007) Heart-type fatty acid-binding protein permits early risk stratification of pulmonary embolism. Eur Heart J 28 (2):224-229

23. Aujesky D et al (2006) Prognostic value of D-dimer in patients with pulmonary embolism. Thromb Haemost 96(4):478-482

24. Lankeit $M$ et al (2008) Growth differentiation factor-15 for prognostic assessment of patients with acute pulmonary embolism. Am J Respir Crit Care Med 177(9):1018-1025

25. Goldhaber SZ (2002) Echocardiography in the management of pulmonary embolism. Ann Intern Med 136(9):691-700

26. Becattini C, Agnelli G (2007) Acute pulmonary embolism: risk stratification in the emergency department. Intern Emerg Med 2 (2):119-129

27. Kasper W et al (1997) Management strategies and determinants of outcome in acute major pulmonary embolism: results of a multicenter registry. J Am Coll Cardiol 30(5):1165-1171

28. Kucher N et al (2005) Prognostic role of echocardiography among patients with acute pulmonary embolism and a systolic arterial pressure of $90 \mathrm{mmHg}$ or higher. Arch Intern Med 165(15):17771781

29. Sukhija R et al (2005) Association of right ventricular dysfunction with in-hospital mortality in patients with acute pulmonary embolism and reduction in mortality in patients with right ventricular dysfunction by pulmonary embolectomy. Am J Cardiol 95(5):695-696

30. Grifoni S et al (2000) Short-term clinical outcome of patients with acute pulmonary embolism, normal blood pressure, and echocardiographic right ventricular dysfunction. Circulation 101 (24):2817-2822

31. Kasper W et al (1986) Echocardiographic findings in patients with proved pulmonary embolism. Am Heart J 112(6):1284-1290

32. Kostrubiec $\mathrm{M}$ et al (2005) Biomarker-based risk assessment model in acute pulmonary embolism. Eur Heart J 26(20):2166-2172

33. Remy-Jardin M et al (2007) Management of suspected acute pulmonary embolism in the era of CT angiography: a statement from the Fleischner Society1. Radiology 245(2):315-329

34. Gibson N, Sohne M, Buller H (2005) Prognostic value of echocardiography and spiral computed tomography in patients with pulmonary embolism. Curr Opin Pulm Med 11(5):380-384

35. van der Meer RW et al (2005) Right ventricular dysfunction and pulmonary obstruction index at helical CT: prediction of clinical 
outcome during 3-month follow-up in patients with acute pulmonary embolism. Radiology 235(3):798-803

36. Kamel E et al (2008) Computed tomographic angiography in acute pulmonary embolism: do we need multiplanar reconstructions to evaluate the right ventricular dysfunction? J Comput Assist Tomogr 32(3):438-443

37. Quiroz R et al (2004) Right ventricular enlargement on chest computed tomography: prognostic role in acute pulmonary embolism. Circulation 109(20):2401-2404

38. Schoepf UJ et al (2004) Right ventricular enlargement on chest computed tomography: a predictor of early death in acute pulmonary embolism. Circulation 110(20):3276-3280

39. Wittenberg R et al (2011) Comparison of automated 4-chamber cardiac views versus axial views for measuring right ventricular enlargement in patients with suspected pulmonary embolism. Eur J Radiol. doi:10.1016/j.ejrad.2011.01.041

40. Masotti L et al (2009) Prognostic stratification of acute pulmonary embolism: focus on clinical aspects, imaging, and biomarkers. Vasc Health Risk Manag 5(4):567-575

41. Ghaye B et al (2006) Severe pulmonary embolism: pulmonary artery clot load scores and cardiovascular parameters as predictors of mortality. Radiology 239(3):884-891

42. Ghuysen A et al (2005) Computed tomographic pulmonary angiography and prognostic significance in patients with acute pulmonary embolism. Thorax 60(11):956-961

43. Zhao D-J et al (2010) Cardiovascular parameters to assess the severity of acute pulmonary embolism with computed tomography. Acta Radiol 51(4):413-419

44. Araoz PA et al (2003) Helical CT pulmonary angiography predictors of in-hospital morbidity and mortality in patients with acute pulmonary embolism. J Thorac Imaging 18(4):207-216

45. Ghaye B et al (2006) Can CT pulmonary angiography allow assessment of severity and prognosis in patients presenting with pulmonary embolism? What the radiologist needs to know. Radiographics 26(1):23-39

46. Nural MS et al (2009) Computed tomographic pulmonary angiography in the assessment of severity of acute pulmonary embolism and right ventricular dysfunction. Acta Radiol 50(6):629-637

47. Bankier AA et al (1997) Severity assessment of acute pulmonary embolism with spiral CT: evaluation of two modified angiographic scores and comparison with clinical data. J Thorac Imaging 12 (2):150-158
48. Mastora I et al (2003) Severity of acute pulmonary embolism: evaluation of a new spiral CT angiographic score in correlation with echocardiographic data. Eur Radiol 13(1):29-35

49. Qanadli SD et al (2001) New CT index to quantify arterial obstruction in pulmonary embolism: comparison with angiographic index and echocardiography. Am J Roentgenol 176(6):1415-1420

50. Bazeed MF et al (2010) Prediction of pulmonary embolism outcome and severity by computed tomography. Acta Radiol 51 (3):271-276

51. Coche E et al (2005) Evaluation of biventricular ejection fraction with ECG-gated 16-slice CT: preliminary findings in acute pulmonary embolism in comparison with radionuclide ventriculography. Eur Radiol 15(7):1432-1440

52. Remy-Jardin M et al (2006) MDCT of right ventricular function: impact of methodologic approach in estimation of right ventricular ejection fraction, part 2. Am J Roentgenol 187 (6): $1605-1609$

53. Sommer WH et al (2010) Saving dose in triple-rule-out computed tomography examination using a high-pitch dual spiral technique. Invest Radiol 45(2):64-71

54. Hoey ETD et al (2009) Dual-energy CT pulmonary angiography: a novel technique for assessing acute and chronic pulmonary thromboembolism. Clin Radiol 64(4):414-419

55. Nikolaou $\mathrm{K}$ et al (2010) Diagnosing pulmonary embolism: new computed tomography applications. J Thorac Imaging 25(2):151-160

56. Lee $\mathrm{C}$ et al (2010) Evaluation of computer-aided detection and dual energy software in detection of peripheral pulmonary embolism on dual-energy pulmonary CT angiography. European Radiology $1-9$

57. Engelke C et al (2010) Does computer-assisted detection of pulmonary emboli enhance severity assessment and risk stratification in acute pulmonary embolism? Clin Radiol 65(2):137-144

58. Jiménez D, Yusan RD (2008) Prognostic models for selecting patients with acute pulmonary embolism for initial outpatient therapy. Curr Opin Pulm Med 14(5):414-421

59. Kreit JW (2004) The impact of right ventricular dysfunction on the prognosis and therapy of normotensive patients with pulmonary embolism. Chest 125(4):1539-1545

60. Leacche $M$ et al (2005) Modern surgical treatment of massive pulmonary embolism: Results in 47 consecutive patients after rapid diagnosis and aggressive surgical approach. J Thorac Cardiovasc Surg 129(5):1018-1023 